\title{
Status of fetal tissue transplantation for the treatment of advanced Parkinson disease
}

\author{
Paul E. Greene, M.D., and Stanley Fahn, M.D. \\ Department of Neurology, Columbia University College of Physicians and Surgeons, New York, New \\ York
}

\begin{abstract}
In the first double-blind, placebo-controlled randomized study of fetal tissue transplantation for the treatment of patients with advanced Parkinson disease (PD), investigators found that implanted dopaminergic tissue can produce measurable improvement in young PD in the absence of medication (that is, the "off" state). The results of the study, however, also highlighted several serious limitations of transplantation. In the group of older patients in the study (in the typical age range of individuals afflicted with PD) no improvement was derived from the implant despite positron emission tomography-documented scan evidence that the graft survived and produced dopamine. Patients in the study were selected because they experienced motor fluctuations, and the transplant did not improve dyskinesias or the time required to remain "on" medication for any subgroup of patients, including young patients. Five of 33 implant-treated patients developed involuntary movements (dyskinesias or dystonia) that could not be eliminated by reducing antiparkinsonian medications. These included four patients with the best responses to transplantation. Finally, some sham-operated patients experienced a dramatic placebo effect lasting at least 1 year, which justified the controversial sham surgery. The authors believe that these problems must be solved before fetal tissue transplantation can be considered a therapeutic option for PD.
\end{abstract}

\section{KEY WORDS • Parkinson disease • dyskinesia • fetal cell transplantion}

There has been considerable interest within the medical and scientific communities about the potential for cell grafting in the treatment of PD. We recently completed a double-blind placebo-controlled study of fetal mesencephalic dopaminergic tissue implanted into the putamen for the treatment of advanced PD. ${ }^{2}$ In the wake of a long series of encouraging open-label studies of fetal tissue transplantation, the outcome of our study, although not entirely negative, was disappointing. This has generated controversy about the relevance of the study to future transplantation efforts. We would like to summarize our interpretation of the results of the study.

\section{COMMENTARY ON RESULTS OF FETAL TISSUE IMPLANT STUDY}

The study was a collaboration between the University of Colorado, Columbia-Presbyterian Medical Center, and North Shore University Hospital. We enrolled 40 patients in whom PD had been present at least 7 years and in

Abbreviations used in this paper: $\mathrm{PD}=$ Parkinson disease; PET $=$ positron emission tomography; UPDRS = Unified PD Rating Scale. whom disabling motor symptoms occurred despite optimal pharmacological management. Patients were stratified in advance into two groups: those older than 60 and those 60 years of age or younger at the time of recruitment. Patients were randomized to undergo fetal tissue implantation or sham surgery. A battery of tests was performed at baseline and during the year-long double-blind phase, including off- and on-medication inpatient UPDRS, timed motor tests, and home diaries of drug dosages of off and on states. Patients underwent a blinded fluorodopa PET scanning at baseline and at 1 year after surgery. After the "blind" was broken at 1 year, patients who had undergone the sham procedure had the option of undergoing implantation immediately. One patient who received the implantation during the double-blind phase died 8 months after surgery (motor vehicle accident) and 14 of 20 sham-treated surgery patients returned to undergo the actual fetal tissue implantation. Thus, we gathered double-blind data in 19 patients obtained for the 1-year period after implantation as well as open-label follow-up data these 19 in addition to 14 others (total 33 patients). All patients who had fetal tissue implants continued home and hospital evaluations every 6 months, and PET scans were obtained every 2 years. Details of fetal tissue processing and complete study methods have been previously published. ${ }^{2}$ 
There was no statistically significant difference between implant- and sham-treated groups in the primary outcome, a subjective comparative global rating determined 1 year after surgery. Placebo-treated patients rated their status as improved by a mean of +0.3 of a possible \pm 3 points, whereas implant-treated patients rated their status as improved by a mean +0.6 of \pm 3 points. Prior to the study, the investigators considered a change of 1.5 of 3 points as clinically meaningful. There were no significant differences in any outcome measures while in the on-medication state for the group as a whole or for either younger or older patients. The total mean UPDRS off-medication score improved more in the implant-treated group (preoperative score of 58 to postoperative score of 50) than in the sham group (from 66 to 63, respectively) and this was of borderline significance $(p=0.055)$. In addition, other off-state measures (total motor, bradykinesia, and rigidity subscores of the UPDRS) and Schwab and England disability scale scores were significantly more improved in the implant-treated than the sham-treated group (p value range 0.00001-0.017). Significant improvement in these scores, however, occurred only in the group of younger patients, who improved markedly in the off-medication UPDRS total score (60-45), UPDRS motor subscore (from 38-26), rigidity and bradykinesia subscores, and Schwab and England Scale scores compared with the placebo-treated group ( $\mathrm{p}$ value range 0.00030.02 ). No improved tremor in the off state was evident in either age group. Walking did not improve significantly, based on examination or history, and walking and balance actually worsened in the group of patients older than 60 years of age $(p=0.033)$. There were no significant differences between implant- and sham-treated patients in either age group in terms of the amount of time off medication, on medication, or on medication without experiencing dyskinesias.

In 12 of 19 implant-treated patients PET scanning demonstrated improvement $(\geq 0.2)$ in striatal/occipital ratio on at least one side of the brain. In sham-treated operated patients no improvement in striatal/occipital ratio of this magnitude was shown. This improvement, however, did not correlate with improvement in parkinsonian ratings in the off-medication state because the older group had a mean PET-documented improvement similar to that observed in the younger group but did not have a comparable improvement in UPDRS scores.

Of considerable concern to us were the five of the 33 implant-treated patients in whom severe dyskinesias and/ or dystonia developed in the absence or near absence of levodopa and dopamine agonists (that is, "runaway" dyskinesias). Two of these patients were able to stop all antiparkinsonian medications and the involuntary movements persisted. These patients had experienced among the most dramatic improvements. Despite multiple attempts to adjust medications and deep brain stimulation in the globus pallidus in three cases, the involuntary movements continued to be troublesome.

\section{QUESTIONS RAISED BY THE FETAL TISSUE IMPLANT STUDY}

We would like to address in detail several of the questions raised by this study.

\section{Why was no Improvement Seen in Medication-Related Motor Fluctuations Despite Reports of Such Improvement in Open-Label Studies?}

The patients in our study were chosen because they experienced a significant response to levodopa but troublesome motor fluctuations that defied management by current methods. The most common problem was disabling dyskinesias even in patients receiving doses of medication sufficient to provide a reasonable duration of acceptable motor functioning, although some patients also suffered off-medication freezing, off-medication anxiety, and a variety of other problems. Even in the subgroup of younger patients, improvement in scores while off medication, in the absence of levodopa, was not sufficient to produce an improvement in the quality of life for the group as a whole. Some individual patients did appear to exhibit improvement in motor fluctuations. This will not be helpful in the clinical setting, however, unless such patients can be identified in advance. We should note that, at initiation of the study, ropinirole and entacapone were not commercially available in the United States. It is possible that these medications might have affected recruitment, but it is unlikely that they would have affected the outcome in a major way. The absence of improvement in time on medication or time on medication with dyskinesias is a major limitation of fetal tissue transplantation in our study. It is possible that an increase in the amount of surviving dopaminergic tissue might overcome this limitation; however, this might also predispose patients to runaway dyskinesias (see following section). Some authors have argued that deficiencies in our technique accounted for the lack of improvement of motor fluctuations in this study. At least six groups have reported improvement in motor fluctuations after fetal tissue implantation in open-label studies. ${ }^{1,5-8,11}$ There is no way to know, however, whether improvements reported by those groups was due to placebo effect. Only additional placebo-controlled studies can settle this question.

\section{Why was Clinical Improvement Absent in Older Patients Despite Improved PET Results?}

We do not know why graft survival, as gauged by fluorodopa PET, did not correlate with clinical improvement in the off-medication state. It is disappointing that clinical improvement on average did not occur in patients in the typical age range for PD, (age $>60$ years) despite apparent graft survival and dopamine production. Once again, this has been attributed to peculiarities in tissue processing or surgical technique in this study (tissue was cultured prior to implantation; no immunosuppression was used, and a novel anterior-posterior surgical approach was used instead of the more common coronal approach). Although it is easy to generate plausible hypotheses, it remains to be shown why these technical variances should affect younger and older patients differently. Examination of the open-label literature does not clearly indicate whether other investigators found similar problems. It remains for the authors of future controlled, agestratified studies to determine whether this problem is associated with other techniques of fetal tissue processing and implantation. 


\section{Did Runaway Dyskinesias Result From Focal Excess Dopamine Production?}

Five of our patients developed dyskinesias and dystonia that persisted in the absence or near absence of levodopa and other antiparkinsonian medications (some patients were unable to stop medications completely because of residual parkinsonian symptoms). These involuntary movements were disabling in four patients, in whom the best fetal tissue transplantation-induced outcomes were achieved, making these dyskinesias a significant concern despite the relatively small number of patients involved. These patients all experienced substantial clinical improvement, and fluorodopa PET revealed a larger increase in signal than in those in whom runaway dyskinesias did not develop. ${ }^{9}$ This suggests that the dyskinesias resulted from excess graft-related dopamine production. Although no larger than normal concentrations of dopamine for an entire putamen occurred in any transplant-treated patient, it is likely, based on autopsy and PET studies, that regions within the putamen may have received large concentrations of dopamine. 3,9 There is one other report, of off-medication dyskinesias in 14 transplanted patients, in which the authors concluded that excess dopamine was not likely the cause of dyskinesias ${ }^{4}$ In that study patients were included if dyskinesias were present in the morning before taking their usual dosage of medications. Dyskinesias seemed to correlate with the severity of preoperative dopamine deficit demonstrated on fluorodopa PET scans, suggesting that striatal denervation, not graft dopamine production, was the major factor producing off-medication dyskinesias. Clinically troublesome dyskinesias, however, were present in only one patient, and they persisted for at least 9 weeks off levodopa and dopamine agonists. It is not known in most of those cases if the dyskinesias persisted in the absence of antiparkinsonian medications. Thus, these cases are not comparable with our cases with runaway dyskinesias. If graft tissue-produced dopamine does cause runaway dyskinesias, attempts to increase graft-produced dopamine (for example, by using trophic factors) may induce even more runaway dyskinesias. In addition, this raises the possibility that treatment of PD by stem cell implant procedures, in which it is easier to implant larger number of cells, may also suffer from the problem of runaway dyskinesias.

\section{Was it Ethical to Use Sham Surgery Rather Than Compare Surgery With Best Medical Therapy?}

At the time our study was proposed, there was public discussion about the ethical implication of involving patients in a sham surgery procedure. In our study, the sham procedure consisted of twist drill holes made in the forehead without needle passes through the dura into the brain. We argued that the risks of sham surgery had to be weighed against the greater risks of performing a procedure that was mistakenly believed to be useful because of placebo effects. If not for concern about placebo effects, it would be reasonable to rely on comparisons between patients randomized to surgery or best medical therapy. We believe that sham surgery is ethical if the placebo effect (1 year after transplantation) is sufficient to affect the outcome of the study. In fact, there was no mean change in
UPDRS score in sham-treated patients. This does not, however, mean that placebo effect did not influence the outcome of the study. The mean improvement in implanttreated patients was $32 \%$ in the off-medication motor UPDRS score. There were four placebo-treated patients who experienced comparable improvements in off-medication motor UPDRS scores, ranging from 25 to $31 \%$, and another patient with a $23 \%$ improvement in off-medication motor UPDRS score. Thus, the placebo effect does increase the variability in outcome, which influences statistical comparison between transplant- and sham-treated groups. The placebo-induced improvement in our study was measured 1 year after sham surgery and thus was very long lasting. Additionally, relatively little is known about the factors that influence the magnitude of the placebo effect. It is not surprising, therefore, that the authors of another placebo-controlled study of tissue implants (using porcine fetal mesencephalic tissue) did find a statistically significant improvement in the placebo-treated group. ${ }^{10}$

\section{CONCLUSIONS}

The results of this study indicate that fetal mesencephalic grafts implanted using the aforementioned techniques and without immunosuppression, can be safely performed. In the majority of cases the grafts survived and produced dopamine, as reflected by improved in fluorodopa PET signal. The grafts were also associated with improved motor function in the off-medication state in younger patients but not in patients older than age 60 years, the typical age range for PD. Motor fluctuations in response to medications were the motivation for seeking surgical therapy in our patients, and these did not improve. Finally, some patients, particularly those with the most dramatic improvement, developed disabling dyskinesias and dystonia persisting in the absence of medications, probably caused by the fetal grafts. Examination of PET studies obtained in the patients with dyskinesias suggested that focal excess graft-produced dopamine caused the troublesome involuntary movements. We cannot determine from this study whether alternative techniques of fetal tissue processing or implantation will produce greater benefit without uncontrollable dyskinesias or whether there are some identifiable patients with PD (such as those with early- or mild-stage disease) who might fare better with fetal tissue grafting. Only further controlled studies can answer these questions.

\section{References}

1. Defer GL, Geny C, Ricolfi F, et al: Long-term outcome of unilaterally transplanted parkinsonian patients. I. Clinical approach. Brain 119:41-50, 1996

2. Freed CR, Greene PE, Breeze RE, et al: Transplantation of embryonic dopamine neurons for sever Parkinson's disease. $\mathbf{N}$ Engl J Med 344:710-719, 2001

3. Freed CR, Trojanowski JZ, Galvin JE, et al: Embryonic dopamine cells cultured as strands show long term survival without immunosuppression in a patient with advanced Parkinson's disease. Soc Neurosci Abstracts 23:682, 1997 (Abstract)

4. Hagell P, Piccini P, Bjorklund A, et al: Dyskinesias following neural transplantation in Parkinson's disease. Nat Neurosci 5: 627-628, 2002 
5. Hauser RA, Freeman TB, Snow BJ, et al: Long-term evaluation of bilateral fetal nigral transplantation in Parkinson disease. Arch Neurol 56:179-187, 1999

6. Henderson BT, Clough CG, Hughes RC, et al: Implantation of human fetal ventral mesencephalon to the right caudate nucleus in advanced Parkinson's disease. Arch Neurol 48:822-827, 1991

7. Kopyov OV, Jacques DS, Lieberman A, et al: Outcome following intrastriatal fetal mesencephalic grafts for Parkinson's patients is directly related to the volume of grafted tissue. Exp Neurol 146:536-545, 1997

8. Lopez-Lozano JJ, Bravo G, Brera B, et al: Long-term improvement in patients with severe Parkinson's disease after implantation of fetal ventral mesencephalic tissue in a cavity of the caudate nucleus: 5-year follow up in 10 patients. Clinica Puerta de Hierro Neural Transplantation Group. J Neurosurg 86: 931-942, 1997

9. Ma Y, Dhawan V, Fukuda M, et al: Dyskinesias following dopamine cell implantation for Parkinson's disease are related to increases in putamen FDOPA uptake. Soc Neurosci Abstr: Program No. 9.1, 2001 (Abstract)

10. Watts RL, Freeman TL, Hauser RA, et al: A double-blind, randomized, controlled, multicenter clinical trial of the safety and efficacy of stereotaxic intrastriatal implantation of fetal porcine ventral mesencephalic tissue (Neurocell'-PD) vs. imitation surgery in patients with Parkinson's disease (PD). Parkinsonism Relat Disord 7 (Suppl):S87, 2001

11. Widner H, Tetrud J, Rehncrona S, et al: Bilateral fetal mesencephalic grafting in two patients with Parkinsonism induced by 1-methyl-4-phenyl-1, 2, 3, 6-tetrahydropyridine (MPTP). N Engl J Med 327:1556-1563, 1992

Manuscript received October 17, 2002.

Accepted in final form October 25, 2002.

Address reprint requests to: Paul E. Greene, M.D., Neurological Institute, 710 West 168th Street, New York, New York 10032. email: pgreene@neuro.columbia.edu. 